\title{
Phase-Variance reduction of high order Stokes lines in Brillouin fiber lasers
}

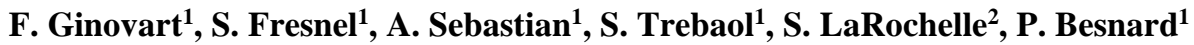 \\ 1. FOTON CNRS UMR 6082, Université de Rennes 1, ENSSAT, Lannion, France \\ 2. COPL, Université Laval, Québec QC, Canada G1V 0A6
}

Brillouin fiber ring lasers have already been extensively studied [1] and results have shown that the generated first order Stokes wave has less intensity and phase noise than the seeded pump. This increase in coherence is attractive for many sensing applications, whether in the field of fiber sensors or remote sensing systems such as LIDAR. Brillouin fiber lasers with several Stokes components can further increase the coherence through a cascading effect, the $1^{\text {st }}$ Stokes component being the pump for the $2^{\text {nd }}$ Stokes order and so on [2]. In this communication, we investigate the noise properties of a multi-Stokes Brillouin fiber laser. We present numerical simulations from a model that is an extension of the usual three-waves model of stimulated Brillouin scattering in agreement with experimental results [2].

The numerical procedure is based on the method of characteristics. Noise is taken into account with a Langevin term describing the random thermal fluctuations of density [3]. The phase noise of the injected pump is simulated through a random walk with a $\delta$-correlated Gaussian noise of zero-mean. Figure 1 shows the output power of the $1^{\text {st }}-4^{\text {th }}$ Stokes components versus the power of the input pump. Figure 1 presents typical simulation results that are in agreement with former theoretical analysis [4] and our experiments. In all plots, the laser-cavity fiber-length is $16 \mathrm{~m}$, the fiber-core diameter is $10 \mu \mathrm{m}$, the Brillouin linewidth is 10 $\mathrm{MHz}$ and Brillouin gain is $7.4610^{-11} \mathrm{~m} / \mathrm{W}$. The pump wavelength is $1550 \mathrm{~nm}$. The laser-clamping of the optical pump is clearly seen as plateaus in the optical power characteristic. When Stokes of order $n$ is clamped, its plateau is directly linked to laser-saturation effect. Meanwhile the increase of the pump power will create photons that lead to lasing effect of the Stokes component of order $n+1$. The results of simulations for phase noise are summarized in figure 2. The variance of the phase of the input pump and output Stokes waves $\left(1^{\text {st }}\right.$ and $2^{\text {nd }}$ order) defined by $\sigma^{2}(\tau)=\left\langle(\phi(\tau)-\phi(0))^{2}\right\rangle$ [1] was statistically averaged over 1000 realizations. Figure 2 shows that the variances are roughly linear in time and that it is reduced by a factor of 1/10 through each successive power transfer between Stokes waves. Thereby linewidths associated to higher orders Stokes components are reduced as previously observed experimentally.

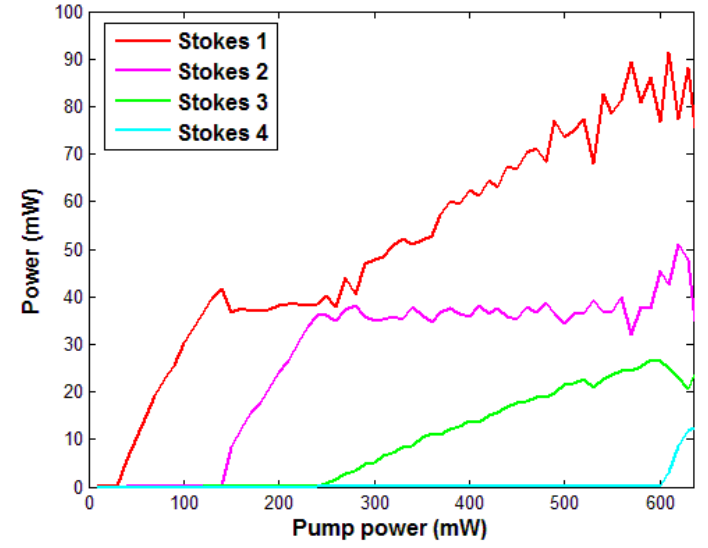

Figure 1: Output Stokes (order 1 to 4 ) power vs injected pump one.
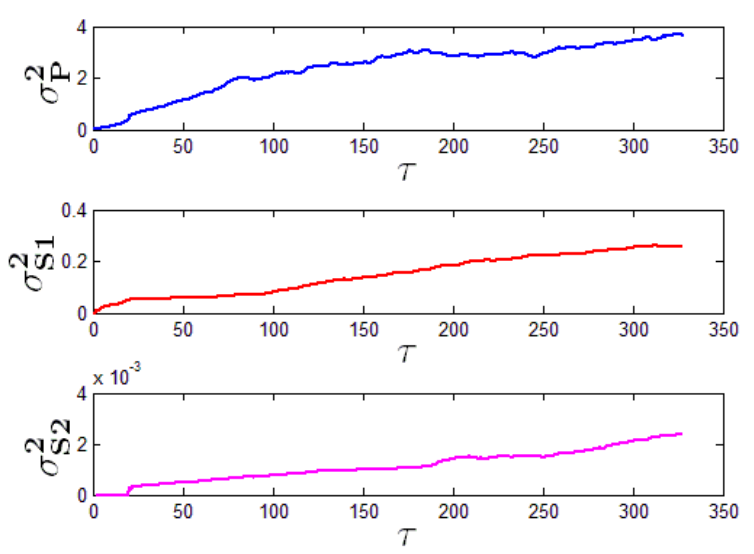

Figure 2: Temporal evolution of the variances of phase for the input pump and output Stokes waves (order 1 and 2) for 1000 realizations.

\section{References}

[1] A. Debut, S. Randoux, and J. Zemmouri, "Linewidth narrowing in Brillouin lasers: Theoretical analysis," Phys. Rev. A - At. Mol. Opt. Phys.,vol. 62, no. 2, pp. 1-4, 2000.

[2] K. H. Tow, Y. Léguillon, S. Fresnel, P. Besnard, L. Brilland, D. Méchin, P. Toupin, and J. Troles, "Toward more coherent sources using a microstructured chalcogenide brillouin fiber laser," IEEE Photonics Technol. Lett., vol. 25, no. 3, pp. 238-241, 2013.

[3] R. W. Boyd, K. Rzaewski, and P. Narum, "Noise initiation of stimulated Brillouin scattering," Phys. Rev. A, vol. 42, no. 9, pp. 55145521,1990 .

[4] K. Toyama, S. Huang, P.-A. Nicati, B. Y. Kim, and H. J. Shaw, "Generation of multiple Stokes waves in a Brillouin fiber ring laeser," Symp A Q. J. Mod. Foreign Lit., pp. 11-14, 1993. 